\title{
Die Schwarze Kirche als Topos der kollektiven Identitätskonstruktion in der deutschen, rumänischen und ungarischen Lyrik der Zwischenkriegszeit
}

\author{
Réka JAKABHÁZI \\ Lekt. Dr., Babeș-Bolyai-Universität Klausenburg. \\ E-Mail: jakabhazireka@gmail.com
}

\begin{abstract}
The Black Church, the largest sacral building in Transylvania, has been given a central role in the local identity narratives. As a historical place of remembrance, it mediates and mobilizes elements of historical knowledge, and at the same time constructs a myth. The article examines how the Black Church in Brasov, one of the most important symbols of the Transylvanian Saxons, is poetically constructed as a place of cultural memory in the German, Romanian and Hungarian poems of the interwar period, how the concrete place is reinterpreted as a space for creating identity, while the ethnic dimension should not be ignored. It examines the question of what symbolic value it has for the German, Romanian and Hungarian populations and how this can be seen from the lyrical texts of the time.
\end{abstract}

Die lyrischen Erinnerungen an die siebenbürgische Geschichte (sei es aus deutscher, rumänischer oder ungarischer Perspektive) bewegen sich häufig zwischen Mythos und Realität und fungieren als identitätsstiftende Faktoren. Wichtig dabei ist zu beobachten, wie die Architektur einer Stadt zur Identitätsbildung der verschiedenen hier lebenden Bewohnergruppen beitragen kann - und wie sich dies in der Literatur widerspiegelt.

In meinem Beitrag habe ich mir vorgenommen, die lyrische Repräsentation des architektonischen Kronstadts, der historischen Bauwerke mit Fokus auf die Schwarze Kirche in der 
dreisprachigen Literatur der Zwischenkriegszeit unter die Lupe zu nehmen. Die Zeitspanne, die gewählt worden ist, ist eine der vielleicht spannendsten in der Geschichte der Stadt: Sie ist geprägt von politischen Umbrüchen und damit verbundenen demografischen Veränderungen.

Um die Veränderungen in der demografischen Konstellation nachvollziehen zu können, soll hier eine Statistik geboten werden:

\begin{tabular}{|c|c|c|c|c|c|}
\hline JAHR & $\begin{array}{l}\text { EINWOHNER- } \\
\text { ZAHL }\end{array}$ & DEUTSCHE & RUMÂNEN & UNGARN & $\begin{array}{c}\text { ANDERE } \\
\text { (Juden, Rroma, } \\
\text { Griechen usw.) }\end{array}$ \\
\hline 1850 & 21.782 & $8.874(40,8 \%)$ & $8.727(40 \%)$ & $2.939(13,4 \%)$ & 2.089 \\
\hline 1890 & 30.739 & $9.578(31,2 \%)$ & $9.758(31,7 \%)$ & $10.441(34 \%)$ & 3.753 \\
\hline 1930 & 59.232 & $13.014(22,0 \%)$ & $19.372(32,7 \%)$ & $23.269(39,3 \%)$ & 2.534 \\
\hline 2011 & 227.961 & $1.079(0,5 \%)$ & $208.757(91,3 \%)$ & $16.172(7,1 \%)$ & 1.953 \\
\hline
\end{tabular}

Wie zu bemerken ist, schrumpfte die deutsche Bevölkerung, die bis zum Anfang des 20. Jahrhunderts die Mehrheit in der Stadt ausmachte, im letzten Jahrhundert dramatisch. ${ }^{1}$

In einer multiethnisch geprägten Region wird den historischen Bauten eine symbolische Bedeutung zugeschrieben, sie tragen zur kollektiven Identitätskonstruktion bei. Im vorliegenden Beitrag wird der Fokus auf die Schwarze Kirche als repräsentativstes Architekturdenkmal der Stadt gelegt, da diese im kulturellen Raum von Kronstadt als kollektiver Gedächtnisraum - gleichwohl ob aus deutscher, rumänischer oder ungarischer Perspektive - eine besondere identitätsstiftende Bedeutung erhalten hat. Deswegen ist es bei der Beschäftigung mit lyrischen Texten mit Kronstadt-Bezug unumgänglich, dieses Wahrzeichen der Stadt vorrangig zu untersuchen und seine Funktion als

Vgl. Volkszählung von 1850, 1890, 1930 und 2011. In: Institutul Național de Statistică: Recensământul populației și a locuințelor. Internet: http://www.recensamantromania.ro/ [Zugriff: 07.02.2019] 
Erinnerungsort ${ }^{2} \mathrm{zu}$ deuten - und als solches vermittelt und mobilisiert es Elemente historischer Kenntnisse; darüber hinaus wird zugleich ein Mythos konstruiert.

Die Schwarze Kirche als Topos der Selbstbestimmung der Siebenbürger Sachsen ist in den deutschsprachigen KronstadtGedichten der ersten Hälfte des 20. Jahrhunderts ein immer wiederkehrender Bezugspunkt bzw. Bestimmungsfaktor für Identität und Selbstverortung. Sie ist und war schon immer ein besonderer Ereignis- und Erinnerungsort, an dem sich das Identitätsbewusstsein und die historische und kulturelle Selbstverortung der Kronstädter verdichtet hat. Als „Dingsymbol kollektiver Identität" nimmt sie für die siebenbürgisch-sächsische Gemeinschaft laut Peter Motzan ${ }^{3}$ die „Funktion eines Identifikators" an, was vielfältig begründet wird: mit der geografischen Lage im Zentrum der Stadt und Rumäniens, ihren Dimensionen, ihrer bewegenden Geschichte und der Rolle, die sie in der Reformationszeit gespielt hat, ihrer Funktion als Kulturstätte und nicht zuletzt durch die traumatische Erfahrung „von Terror und Justizwillkür, die unter der kommunistischen Diktatur, im ‘Schwarzen-Kirche-Prozess` (1958) durchlitten wurde". ${ }^{4}$

Sie figuriert als Emblem der Gruppen-Kohäsion und GruppenSolidarität, als Stätte der Selbstfindung, als Nachweis einer bleibenden kulturellen Leistung und als Kronzeuge der Überlebenskraft und Daseinskontinuität der Siebenbürger Sachsen in der angestammten Heimat. $^{5}$

2 Im vorliegenden Beitrag wird der Begriff imSinne von Pierre Nora (,lieux de mémoire”) verstanden. Vgl. Nora, Pierre: Zwischen Geschichte und Gedächtnis. Frankfurt am Main: Fischer 1998.

3 Motzan, Peter: Dingsymbol kollektiver Identität. Die Schwarze Kirche als „Gegenstand“ der siebenbürgisch-deutschen Literatur (1919-1944). In: 50 Jahre Südostdeutsches Kulturwerk - Südostdeutsche Vierteljahresblätter 1951-2001. München 2001, S. 55-64.

4 Ebd. S. 55.

5 Ebd. S. 63. 
Albert Weber, der der Schwarzen Kirche ebenfalls eine umfangreiche Studie ${ }^{6}$ widmet, präsentiert diese als religiösen Ereignisund Erinnerungsort, der „die Beständigkeit, Entschlossenheit und gemeinschaftliche Solidarität symbolisiert".? Als Erinnerungsort kann somit die Schwarze Kirche zugleich als topografisch begrenzte Stätte (Sakralbau) und als geschichtliches Ereignis (nach dem vernichtenden Stadtbrand im Jahre 1689 neu errichtete und dadurch zum Symbol der Beständigkeit gewordene Festung der Sachsen) gedeutet werden, die aber auch zum kulturellen Gedächtnis anderer hier lebenden Nationen gehört (als Bollwerk des Christentums, in der Erinnerung an den osmanischen Ansturm im 16. Jahrhundert). Darüber hinaus wird sie von den verschiedenen Gruppen auch verschiedenartig gewertet: Neben der symbolischen Schutzrolle, die sie für die Siebenbürger Sachsen spielt, trägt sie auch eine weitere wichtige Bedeutung für die Kronstädter, nämlich ,die wirtschaftliche Überlegenheit gegenüber Hermannstadt, dem politischen und kirchlichen Zentrum der Siebenbürger Sachsen" ${ }^{\text {"8 }}$.

Albert Weberweist darauf hin, dass die Schwarze Kirche nicht nur für die Sachsen (die heute infolge der Auswanderung größtenteils in Deutschland leben) ein „bleibendes Zeugnis ihrer Kultur und Geschichte in der alten Heimat" darstellt, sondern, ,als ein solches dient es auch anderen Bevölkerungsgruppen als Erinnerungsort für die Siebenbürger Sachsen". ${ }^{9}$ Die architektonische Bedeutung der Schwarzen Kirche wird von der rumänischen Bevölkerung gewürdigt und allmählich wird sie als Teil des nationalen Kulturerbes verstanden. Außerdem ist die

6 Weber, Albert: Die Schwarze Kirche in Kronstadt. In: Joachim Bahlcke; Stefan Rohdewald; Thomas Wünsch(Hgg.): Religiöse Erinnerungsorte in Ostmitteleuropa: Konstitution und Konkurrenz im nationen- und epochenübergreifenden Zugriff. Berlin: Akademie Verlag 2013, S. 302-312., hier S. 302.

7 Ebd. S. 302.

8 Ebd.

9 Ebd. 
Schwarze Kirche im kulturellen Gedächtnis der Region (gleichwohl welcher Ethnie) fest als Schutzwall Europas verankert:

Hier erscheint in der heutigen Erinnerung ansatzweise das in Südosteuropahäufigeranzutreffende Narrativ des antemurale Christianitatis, jedoch umgestaltet und mit einer fast durchweg positiven Aussage: Kronstadt habe wichtige, opferreiche Abwehrleistungen gegen die expandierenden Osmanen vollbracht, womit Westeuropa geschützt wurde und sich leichter entwickeln konnte. ${ }^{10}$

Als eines der bekanntesten Kronstadt-Gedichte, die die Schwarze Kirche thematisieren, ist Trude Geißler-Nußbächers An die Schwarze Kirche in Kronstadt ${ }^{11} \mathrm{zu}$ nennen.

\section{An die Schwarze Kirche in Kronstadt}

Kirche, in deren Schatten unser Haus gestanden,

War nicht dein Dach wie ein Zelt unterm Himmel gespannt?

All die langen Jahre gab es mir Schutz

Auch ferne von dir in einem andern Land.

Groß ragt es vor dem großen Berg,

Wie er vor dem hohen Himmel ragt -

Einem Lied gleich blieb dies in mir

Und hält in der Fremde das Heimweh wach.

Und nun - alterst du, alte Kirche,

Und es bröckelt dein Stein zu Sand.

Einst schütztest du uns, jetzt schützen wir dich -

Gott gab uns dazu den Sinn und die Hand.

$\mathrm{Da}$ du weiter die Wache hältst im Tal,

Es überschirmend mit deinem Dach,

Daß der Himmel und der Berg nicht einmal

- Ohne Antwort zu finden dir fragen nach.

Die Schwarze Kirche wird hier eindeutig als Symbol der Heimat gewertet, als Schutz, was mit der Metapher des Daches

${ }^{10}$ Weber, S. 305.

${ }^{11}$ In: Roth, Herman; Krasser, Harald (Hgg.): Herz der Heimat. Deutsche Lyrik aus Siebenbürgen. München: Albert Langen Georg Müller Verlag 1937, S. 25. 
visualisiert wird (das das Tal und seine Bewohner überschirmt). Die Wahl der Verben und Funktionsverbgefüge (,schützen“, „hält... wach“, „hält Wache“) untermauern diese Aussage, und die Wiederholung des Verbes ,ragt” innerhalb von zwei Zeilen in Verbindung mit den Adjektiven ,groß”, ,alt” betont die Überlegenheit, Mächtigkeit, und die zentrale Rolle, die die Kirche im Laufe der Jahrhunderte in der Geschichte der Stadt spielte: Sie bietet Schutz und Geborgenheit, bedeutet stets Heimat, die in der Ferne eine zusätzliche Rolle erhält: ,und hält in der Fremde das Heimweh wach." Zu betonen ist jedoch auch die beiderseitige Verantwortung, die im Gedicht angesprochen wird: Nicht nur die Kirche besitzt die Funktion des Wächters, sondern die Sachsen (,wir") haben auch die Pflicht, sie zu beschützen: „Einst schütztest du uns, jetzt schützen wir dich“.

Wie der Text von Trude Geißler-Nußbächer, so ist auch das Gedicht Schwarze Kirche 12 von Maria Scherg eine direkte Anrede an die Schwarze Kirche, die ebenfalls als Symbol des Schutzes (,heilige Wacht“) dargestellt wird.

Eine völlig andere Stimmung vermittelt das Gedicht Kronstadt von Marianne Vincent, in dem durch den Blickwinkel eines Außenseiters ${ }^{13}$ die Schwarze Kirche als „Bekenntnis und als Mahnung" gezeigt wird:

\section{Kronstadt $^{14}$}

Wie ein dunkles Schiff mit dunklen Masten

Stürmst du gegen Bergesbrandung an.

Bange Schickungen sind deines Kieles Lasten

Klingsor ist dein Steuermann.

${ }^{12}$ Scherg, Maria: Schwarze Kirche. In: Dies.: Jahr und Leben. Gedichte. Aus ihrem Nachlass ihren Freunden dargeboten. Kronstadt 1943, S. 29.

${ }^{13}$ Die Dichterin stammt nicht aus dieser Region, die gebürtige Czernowitzerin kam als Schauspielerin nach Siebenbürgen, wo sie als Mitglied des Siebenbürgischen Landestheaters in Hermannstadt tätig war.

${ }^{14}$ In: Kirchliche Blätter der evang. Landeskirche A.B. in Rumänien Nr. 4/32. Jahrgang, 23. Januar 1940. Hermannstadt. S. 41. 
Keine Ruhe ist auf deinen Straßen

Und selbst deine Stille ist noch Ruf.

Sonnenarm sind deine engen Gassen

Die ein strenger Menschenschlag sich schuf.

Wie dich baute alter Meister Planung

Stehst du heute noch in herber Pracht.

Als Bekenntnis und als Mahnung

Hält die Schwarze Kirche Wacht.

Der Vergleich der Stadt mit einem Schiff ist nicht neu: Bereits Meschendörfer hat in seinem Roman Die Stadt im Osten die Schiff-Metapher für ,der kostbarste Schatz, die große Stadtpfarrkirche“"15 angewandt: ,Wo ruht es sich besser als in deinem stolzen steinernen Schiff, das am Fuße des drohenden Berges, inmitten des Getümmels und Hastens Anker geworfen hat und sich majestätisch im Blau des Himmels wiegt?“"16

Das Erwähnen des mittelalterlichen Sängers und Zauberers Klingsor (den die Legende mit Kronstadt verknüpft ${ }^{17}$ ) in der Rolle des Steuermannes im Kronstadt-Schiff trägt zur Verklärung und Mythisierung des Raumes bei, und zugleich wird auf das Historisch-Heroische reflektiert: „Als Bekenntnis und als Mahnung / Hält die Schwarze Kirche Wacht.“"

Ein weiterer Czernowitzer Autor, Immanuel Weissglas, der der Schwarzen Kirche ebenfalls ein Gedicht gewidmet hat, ${ }^{18}$ stellt den Turm der Schwarzen Kirche als ,sternnachbarlich zur Helle“" strebenden Zeitzeugen dar, der, ,in die Höh gequält" als Mahnung verstanden werden kann: Die „Kahlheit des Spitzbogenalls“ wird mit einem, ,schrammgen Strick am Sünderhals“

${ }^{15}$ Vgl. auch: Motzan, S. 58-59.

${ }^{16}$ Meschendörfer, Adolf: Die Stadt im Osten. Hermannstadt: Krafft\&Drotleff 1931, S. 74. Vgl. auch Motzan, S. 58.

${ }^{17}$ Vgl. Roth, Harald: Kronstadt in Siebenbürgen. Eine kleine Stadtgeschichte. Köln - Weimar - Wien: Böhlau 2010, S. 11.

${ }_{18}$ Weissglas, Immanuel: Schwarze Kirche. In: Der Nobiskrug. Gedichte. Bukarest: Kriterion 1972, S. 16. 
verglichen, was dem Gedicht die morbide Atmosphäre einer Hinrichtung verleiht. Dies wird in der vorletzten Zeile von der Darstellung der Kirche als "sündger Stein im Büßerhemde“" verstärkt. Doch das „,ewge Licht“, das ,,von einem Psalm“ genährt wird, kann zur Erlösung führen: „Wie Du, sündger Stein im Büßerhemde, / Erderlösend ragst in keiner Fremde.“. ${ }^{19}$

Wie aus den behandelten Gedichten ersichtlich wurde, wird in der deutschen Lyrik der 20er, 30er und 40er Jahre des 20. Jh.s der Schwarzen Kirche die Rolle des Wächters der siebenbürgisch-sächsischen Kulturwerte beigemessen. So wandelt sie sich zum Erinnerungsraum, der von einer pathetisch dargestellten Vergangenheit, von Mythos und Verklärung beherrscht wird.

Anders als in den deutschen Kronstadt-Gedichten der Zeit wird in der rumänischen bzw. ungarischen Lyrik der Schwarzen Kirche zwar eine bedeutende, doch keineswegs dominante Bedeutung beigemessen: Sie ist mehr als nur Dekor, doch weitaus weniger als Mythos. In den rumänischen Texten wird die symbolische identitätsstiftende Rolle von den für die rumänische Geschichte wichtigen architektonischen Elementen der Stadt übernommen (die Șchei, die Sankt Nikolaus-Kirche), während in den ungarischen lyrischen Texten die Stadt als Ganzes betrachtet wird, es werden keine einzelnen Bauten hervorgehoben, der Erinnerungsraum konstruiert sich aus der Stimmung und den Gefühlen, die das lyrische Ich in der Stadt erlebt.

Im Gedicht Stadt [Citadina] ${ }^{20}$ des rumänischen Dichters I.D. Tudorache wird die Schwarze Kirche zum Beispiel nicht erwähnt, nur indirekt angesprochen: ,wenn die verschlafene Burg sich das schwarze Antlitz wäscht / das von Ferne einem alten Ikonostas gleicht". Wenden wir unsere Aufmerksamkeit diesem Gleichnis zu, so ist unschwer zu bemerken, dass es in der Wortwahl eindeutige sinnstiftende Elemente enthält: Ein

19 Ebd.

${ }^{20}$ In: Gionea, Vasile (Hg.): Antologia scriitorilor din Țara Bârsei. Braşov: Astra 1946, S. 85. 
Ikonostas ist eine mit Ikonen verzierte Trennwand vor dem Altarraum orthodoxer Kirchen; die Tatsache, dass sich die „Burg” und die Schwarze Kirche (der Bezug dazu wird durch das Adjektiv „schwarz” geschaffen) ihr Antlitz waschen, deutet auf eine Art Dekonstruktion und Rekonstruktion des Raumes. Die ,alte Stadt" wird mit Schweigen und Vergänglichkeit assoziiert:

Auf dem Platz schlägt die Uhr tiefen Schweigens, die alte Uhr, mit dem Rathausturm gleichen Alters Und das zweite Mal schlägt das Echo gegen Felsen Die Küsterstimme des traurigen Palastes. ${ }^{21}$

In den Gedichten von Ștefan Baciu Führung für eine ,Tour “ [Ghid de ,Tour"] ${ }^{22}$ bzw. Winterlandschaft in Kronstadt [Peisaj de iarnă la Brașov], ${ }^{23}$ die zwar später als unsere untersuchte Periode geschrieben wurden, die aber das „Kronstadt der Erinnerung" thematisieren (der Dichter verbrachte seine Kindheit und frühe Jugend in den 20er, 30er Jahren in Kronstadt), werden symbolbeladene Räumlichkeiten der Stadt heraufbeschworen: die Schnurstraße, das Rathaus, die Șchei, das Konzerthaus Redoute, der Schwarze Turm, der ,wie ein Symbol über die Stadt ragt“. Die Schwarze Kirche (die ja für die sächsische Bevölkerung der Stadt, wie bereits angedeutet, eine zentrale Bedeutung hat) wird bloß in einer kurzen Auflistung erwähnt, die älteste rumänische Kirche der Stadt (Sankt Nikolaus-Kirche) wird jedoch näher gebracht, indem sie mit dem für die Rumänen wichtigen Dichter und Musiker Anton Pann verknüpft wird, der u.a. die rumänische Nationalhymne Deșteaptă-te române! [Erwache, Rumäne!] vertonte:

Hier ist die Schwarze Kirche. Hier ist das Rathaus.

Dort die Schulerau. Dort ist der Schuler.

${ }^{21}$ Ebd. Übersetzung der Verfasserin.

${ }^{22}$ In: Stoie, Nicolae (Hg.): Brașovul în o sută de poezii. Brașov 2007, S. 48.

${ }^{23}$ Braşovul în o sută de poezii, S. 52. 
Hier ist die Sankt Nikolaus-Kirche

wo auf der Kirchenbank Anton Pann sang. ${ }^{24}$

Der Redoute-Saal, der im kulturellen Gedächtnis der Rumänen von Kronstadt fest verankert ist, da hier die ersten rumänischen Theatervorstellungen aufgeführt wurden (in den 1860er Jahren) wird im Gedicht ebenfalls heraufbeschworen. Somit erfolgt wie auch im Gedicht von Tudorache - eine sinnbildhafte Inbesitznahme des Raumes.

Auch im Gedicht von Lucian Blaga Alte Stadt [Oraș vechi] ${ }^{25}$ wird die vergangene Zeit mit „Nacht", ,Schweigen“, ,,Seufzen“, ,alt", „verschwinden“ und „erloschen“ konnotiert. Zeilen wie „Der Schwarze Turm steht noch / und zählt seine Jahren besiegt" oder „Große Ahnen von einst / zeigen sich für einen Augenblick und verschwinden", beschwören eine versteinerte Welt, in denen nur der ,Wind“ und der ,Regen“" lebendig wirken.

Erst in der zweiten Hälfte des 20. Jahrhunderts, als die rumänische Bevölkerung in Kronstadt zur Mehrheit wurde, gewinnt die Schwarze Kirche eine größere Bedeutung auch im kulturellen Gedächtnis der Rumänen: Im Kulturtourismus spielt sie als Baudenkmal von außerordentlicher nationaler Bedeutung eine wichtige Rolle.

Kronstadt und seine Baudenkmäler fungieren nicht nur für die deutsche und rumänische, sondern ebenfalls für die ungarische Gemeinschaft als Erinnerungsorte. Die Darstellung des Stadtbildes wird demnach in den ungarischen lyrischen Texten zum Teil der Selbstdeutungen und von Deutungen subjektiver wie auch gesellschaftlicher, kultureller und politischer Wandlungsprozesse geprägt. Dies ist vor allem in der Lyrik des gebürtigen Kronstädters Lajos Áprily ersichtlich, dessen dichterisches Werk vor allem von der Landschaft und der umgebenden Natur sowie vom kulturellen Erbe Kronstadts inspiriert wurde.

${ }^{24}$ Braşovul în o sută de poezii, S. 50. Übersetzung der Verfasserin.

${ }^{25}$ Lucian Blaga: Oraș vechi. In: Stoie, Nicolae (Hg.): Brașovul în o sută de poezii. Brașov 2007, S. 23. 
In seinem Gedicht Ein Glas Wein [Egypohárbor] ${ }^{26}$ gedenkt er seiner Kronstädter Vorfahren, von denen mehrere Goldschmiede waren. Der Dichter erstellt eine Parallele zwischen der künstlerischen Feinfühligkeit der Goldschmiedearbeit seiner Ahnen und der von ihm, dem späten Dichter-Nachkommen angestrebten verfeinerten dichterischen Formkunst. Um die besondere klangvolle Musikalität seiner Sprache sichtbar zu machen, werden hier die Verse im Originalzitiert (die vorhandenen Übersetzungen können leider nicht an der Vorlage gemessen werden):

Forma van a versem

egyszerüsorában:

ötvösvoltazősöm

Brassóvárosában. $^{27}$

In diesem, von besonderer Musikalität, besonderem Klang und Rhythmus durchtränkten lyrischen Bekenntnis formuliert Áprily seine Ars Poetica: Seine Verse gelten allesamt als Goldschmiedearbeit, deren Material nicht das Edelmetall, sondern die Sprache ist - die er in Kronstadt erlernt hatte. Die künstlerische Tradition der Vorfahren wird nun durch ihn, den Dichter weitergeführt: Er ist der Reinheit der Form, der edlen Schlichtheit verpflichtet, sein Stil ist wie die Kunststücke seiner Urahnen wohlgemeistert, zugleich spielerisch und erhaben, rein und edel. Im erwähnten Gedicht wird die Parallele jedoch nicht nur auf Formebene gezogen: Ein weiteres wichtiges Merkmal seiner gesamten Dichterkunst ist - laut der allegorischen Andeutungen - auch von seinem Kronstädter (teils sächsischen) Urahn ${ }^{28}$ ge-

${ }^{26}$ In: Áprily Lajos: A láthatatlan írás. Budapest: Révai, 1939, S. 9.

${ }^{27}$ In wortwörtlicher Wiedergabe: „Die einfachen Zeilen/ meines Gedichtes sind wohlgestaltet:/ Meine Vorfahren waren/ Goldschmiede in Kronstadt"“. Um die Musikalität besser wiederzugeben: „Form ist in Gedichtes einfachen Zeilen / Mein Ahn konnte in Kronstadt / Gold und Silber feilen." Übersetzung der Verfasserin.

${ }_{28}$ Áprilys Familie ist teils deutscher, teils ungarischer Herkunft. Sie hießen ursprünglich Jékely (der Name Jeckel/Jickeli bzw. die latinisierte 
erbt worden: das Ineinanderflechten von ethnischer Vielfalt, die Mehrsprachigkeit und Toleranz. Heraufbeschworen werden zugleich die Wappen auf den ,alten Mauern” sowie der fürstliche Kelch von Gabriel Bethlen - sowohl die sächsischen als auch die ungarischen symbolträchtigen Kunstwerke wurden (allegorisch) vom Goldschmied-Vorfahr angefertigt. Durch die Person des Urahns und sein Erbe wird Kronstadt demnach im Gedicht zum Symbolraum der Kunst, der siebenbürgischen Vielvölkerverständigung - wobei zu bemerken ist, dass dieses positive Bild in die Vergangenheit projiziert wird: „Meine Seele füllt sich mit dem Duft der Landschaften / zur Weinlese war ich oft am sonnigen Mieresch / Mostklärend, den besten Wein abwartend, zum edelsten Getränk / bin ich jedoch zurück in die Jahrhunderte gegangen. ${ }^{\text {" }} 29$

Wie aus dem Gedicht deutlich wird, werden hier keine konkreten Orte (Schwarze Kirche usw.) erwähnt; die Raumdarstellung erfolgt durch das Heraufbeschwören des Urahns und der Vergangenheit - dadurch versucht der Dichter das Schicksal eines Siebenbürgers auf den Irrwegen von familiären Legenden, regionalen Mythen und Geschichtsschreibung neu zu interpretieren.

In den Gedichten von Ferenc Szemlér gilt Kronstadt als „Heimat" und „Zuhause“, wie dies aus dem Gedicht Brassó ${ }^{30}$ auch ersichtlich wird. Bereits in der ersten Zeile werden „Türme, alte Mauern, rauschende Wälder" heraufbeschworen, zwischen denen „du dein gekrüppeltes und unsicheres Schicksal lebst“.

Form Jickelius ist in den von Sachsen bewohnten Regionen Siebenbürgens geläufig), seine ersten Gedichte veröffentlichte der junge Autor (Student der Fachrichtung Ungarisch-Deutsch in Klausenburg) noch unter diesem Namen.

29 „Tájakjószagával /telelett a lelkem, /naposMaros-mentén /sokszorszüreteltem. /Mustjátderítettem,/színborátkivártam,/nemesebbetszürni /századokbajártam."

${ }^{30}$ Szemlér Ferenc: Brassó. In: Pásztortüz, 1931. Nov. 22, XVII. Jg, Heft 23, S. 532. 
Die Bauten der Vergangenheit wirken bedrohlich, und doch wird diese Stadt als Zuhause gewertet.

Türme, Mauern, Fabriken und Menschen!...

Wer sieht von ihnen, wer du bist

wer spürt von ihnen, weshalb du gekommen? ${ }^{31}$

Die alte Geschichte der Stadt wird durch das Erwähnen des Hauses mit der Inschrift „1500“ und „einer Kirche“ heraufbeschworen, die ,schon von den Tataren gesehen wurde“. Bemerkenswert ist, dass, obwohl hier eindeutig von der Schwarzen Kirche die Rede ist, sie nicht mit ihrem Eigennamen, sondern mit dem unbestimmten Artikel und einem Appellativ benannt wird - was ihre Wichtigkeit zu mindern scheint. „Morgen wird's vielleicht besser sein / Morgen wird's vielleicht schlechter sein / Oder es wird vielleicht nichts mehr sein" - lautet der pessimistische Ausklang des Gedichtes und: „Die Türme und Mauern / umstehen dich / wie veraltete Hellebardiere" ${ }^{32}$ Dieses „Zuhause“ wird demnach als befremdend, als alt, als verkrüppelt und unsicher, als sterbend dargestellt; nicht die real existierenden topografisch verortbaren Räume von Kronstadt werden als „Heimat” und „Zuhause” empfunden, sondern imaginierte Raumstrukturen; Gefühle, die diese Orte hervorrufen - und die für Szemlér als Wahrzeichen der Stadt fungieren.

Die Schwarze Kirche als Ort des kulturellen Gedächtnisses wird in der Lyrik der Zwischenkriegszeit von den Vertretern der drei Nationen - wie oben dargestellt - jeweils unterschiedlich wahrgenommen, fiktionalisiert und instrumentalisiert: Für die Sachsen repräsentiert sie Schutz und Wache, Bewahrer jahrhundertealter Traditionen, Ankerpunkt für nationale Identifikation bzw. Selbstverortung und zugleich Symbol der Pflicht. In den rumänischen und ungarischen Gedichten verliert die Schwarze Kirche an Relevanz und Bedeutung. Sie tritt in den

${ }^{31}$ Ebd. Übersetzung der Verfasserin.

32 Übersetzung der Verfasserin. 
Hintergrund und macht Raum für neue Interpretationen: Im rumänischen Kontext findet eine De- bzw. Rekonstruktion des Raumes statt, indem für die Rumänen wichtige Orte in den Vordergrund treten; in den ungarischen Gedichten fungiert Kronstadt gleichsam als Heimat bzw. Ort der Zerrissenheit, der Befremdung und die Texte werden der wehmütigen Stimmung des Verlustes untergeordnet. Somit tragen diese Gedichte der drei Literaturen in sehr verschiedenen Weisen zum facettenreichen Bild der kulturellen Eigenart der Region bei.

\section{Literaturverzeichnis}

Institutul Național de Statistică: Recensământul populației și a locuințelor. In: http://www.recensamantromania.ro/ [Zugriff: 07.02.2019]

Motzan, Peter: Dingsymbol kollektiver Identität. Die Schwarze Kirche als „,Gegenstand“ der siebenbürgisch-deutschen Literatur (1919-1944). In: 50 Jahre Südostdeutsches Kulturwerk - Südostdeutsche Vierteljahresblätter 19512001. München 2001, S. 55-64.

Nora, Pierre: Zwischen Geschichte und Gedächtnis. Frankfurt am Main: Fischer 1998.

Roth, Harald: Kronstadt in Siebenbürgen. Eine kleine Stadtgeschichte. Köln - Weimar - Wien: Böhlau 2010.

Weber, Albert: Die Schwarze Kirche in Kronstadt. In: Joachim Bahlcke; Stefan Rohdewald; Thomas Wünsch (Hgg.): Religiöse Erinnerungsorte in Ostmitteleuropa: Konstitution und Konkurrenz im nationen- und epochenübergreifenden Zugriff. Berlin: Akademie Verlag 2013, S. 302-312.

Weitz, Burkhard: Kampflied der Deutschen oder Protest gegen Gewalt? Widersprüchliche Interpretationen von Martin Luthers Choral. In: Chrismon. Das evangelische Magazin. 02.11.2016. Im Internet unter: https://chrismon.evangelisch.de/artikel/2016/32838/ein-feste-burg-ist-unser-gott- 
kampflied-der-deutschen-oder-protest-gegen-irdische-gewalt [Zugriff 7.02.2019]

\section{Gedichte:}

Áprily Lajos: Egy pohár bor. In: Ders.: A láthatatlanírás. Budapest: Révai, 1939, S. 9.

Baciu, Ștefan: Ghid de „Tour“. In: Stoie, Nicolae (Hg.): Braşovul în o sută de poezii. Brașov 2007, S.48.

Baciu, Ștefan: Peisaj de iarnă la Brașov. In: Stoie, Nicolae (Hg.): Braşovul în o sută de poezii. Braşov 2007, S.52.

Blaga, Lucian: Oraș vechi. In: Stoie, Nicolae (Hg.): Braşovul in o sută de poezii. Brașov 2007, S. 23.

Geißler-Nußbächer, Trude: An die Schwarze Kirche in Kronstadt. In: Roth, Herman; Krasser, Harald (Hgg.): Herz der Heimat. Deutsche Lyrik aus Siebenbürgen. München: Albert Langen Georg Müller Verlag 1937, S. 25.

Scherg, Maria: Schwarze Kirche. In: Dies.: Jahr und Leben. Gedichte. Aus ihrem Nachlass ihren Freunden dargeboten. Kronstadt 1943, S. 29.

Szemlér Ferenc: Brassó. In: Pásztortüz, 1931. Nov. 22, XVII. Jg, Heft 23, S. 532.

Tudorache, I.D.: Citadina. In: Gionea, Vasile (Hg.): Antologia scriitorilor din Țara Bârsei. Brașov: Astra 1946, S. 85.

Vincent, Marianne: Kronstadt. In: Kirchliche Blätter der evang.

Landeskirche A.B. in Rumänien Nr.4/32. Jahrgang, 23. Januar 1940. Hermannstadt. S. 41.

Weissglas, Immanuel: Schwarze Kirche. In: Der Nobiskrug.

Gedichte. Bukarest: Kriterion 1972, S.16. 